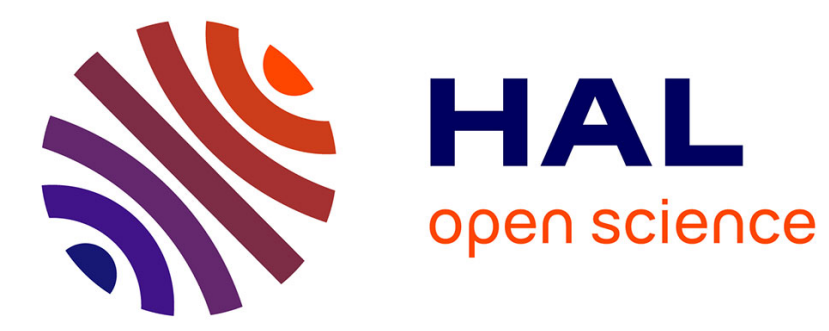

\title{
Network games under strategic complementarities
}

Mohamed Moez Belhaj, Yann Bramoullé, Frédéric Deroïan

\section{To cite this version:}

Mohamed Moez Belhaj, Yann Bramoullé, Frédéric Deroïan. Network games under strategic complementarities. Games and Economic Behavior, 2014, 88 (C), pp.310-319. 10.1016/j.geb.2014.10.009 . hal-01474250

\section{HAL Id: hal-01474250 \\ https: / / hal-amu.archives-ouvertes.fr/hal-01474250}

Submitted on 29 Nov 2018

HAL is a multi-disciplinary open access archive for the deposit and dissemination of scientific research documents, whether they are published or not. The documents may come from teaching and research institutions in France or abroad, or from public or private research centers.
L'archive ouverte pluridisciplinaire HAL, est destinée au dépôt et à la diffusion de documents scientifiques de niveau recherche, publiés ou non, émanant des établissements d'enseignement et de recherche français ou étrangers, des laboratoires publics ou privés. 


\title{
Network games under strategic complementarities
}

\author{
Mohamed Belhaj ${ }^{\mathrm{a}}$, Yann Bramoullé ${ }^{\mathrm{b}, *}$, Frédéric Deroïan ${ }^{\mathrm{b}, 1}$ \\ a Centrale Marseille (Aix-Marseille School of Economics), CNRS \& EHESS \\ ${ }^{\mathrm{b}}$ Aix-Marseille University (Aix-Marseille School of Economics), CNRS \& EHESS
}

Keywords:

Network games

Strategic complementarities

Centrality

Uniqueness Interdependence

\begin{abstract}
A B S T R A C T
We study network games under strategic complementarities. Agents are embedded in a fixed network. They choose a positive, continuous action and interact with their network neighbors. Interactions are positive and actions are bounded from above. We first derive new sufficient conditions for uniqueness, covering all concave as well as some non-concave best responses. We then study the relationship between position and action and identify situations where a more central agent always plays a higher action in equilibrium. We finally analyze comparative statics. We show that a shock may not propagate throughout the entire network and uncover a general pattern of decreasing interdependence.
\end{abstract}

\section{Introduction}

In this paper, we study network games under strategic complementarities. Agents are embedded in a fixed network and choose a positive, continuous action. We assume that actions are bounded from above. Agents play a game of strategic complements with their network neighbors. An agent's action is strictly increasing in her neighbors' actions until it reaches its upper bound. This may capture, for instance, peers' influence in criminal activity or arms race between countries. ${ }^{2}$ We do not impose further assumptions on the shape of the best response. It could be linear, concave, convex or of changing curvature below the upper bound. Our main objective is to analyze the Nash equilibria of these network games.

We have three main contributions. First, we derive new sufficient conditions for uniqueness. Our results provide the first application of a general principle of localized contraction. In supermodular games, contraction of the iterated best response for ordered profiles lying between the extremal equilibria guarantees uniqueness. We show how to apply this principle under various curvature assumptions on the best responses. We find that localized contraction always holds under concavity. Uniqueness in that case follows from the results of Kennan (2001). Our approach provides an alternative proof based on very different premises. Under convexity, we prove that localized contraction holds when the best response is not too convex. And we obtain a sufficient uniqueness condition for arbitrary best responses.

Second, we study the relationship between network position and action in equilibrium. We first show that more central agents may play a lower action. We then identify two situations where action is unambiguously related to position. An agent

\footnotetext{
* Corresponding author.

E-mail address: yann.bramoulle@univ-amu.fr (Y. Bramoullé).

1 We thank Federico Echenique, Matt Jackson, three anonymous referees and participants of the 2012 Coalition Theory Network and the 2013 Journées Louis-André Gérard-Varet for helpful comments and discussions.

2 We discuss these two applications in more detail in Section 2 below.
} 
who has fewer neighbors than another in the sense of set inclusion necessarily plays a lower action in any equilibrium. When the graph is symmetric and structured around a geometric center, like the line, action is aligned with centrality in extremal equilibria. These results hold for any curvature of the best response below the upper bound.

Third, we look at comparative statics. Classical results from the theory of supermodular games tell us that action in an extremal equilibrium is weakly increasing in actions in isolation, upper bounds and link strengths. We then study how a shock propagates throughout the network. We characterize precisely who ends up being affected by a positive shock on one agent. The presence and positions of bridges between communities turn out to be critical. When bridging agents reach their upper bounds, they stop transmitting influence across different parts of the networks and interdependence may be severely reduced.

Our analysis contributes to the literature on games played on fixed networks. A large part of this literature to date has studied games with linear best responses. ${ }^{3}$ Ballester et al. (2006) study network games under linear best responses and small network effects. They find that the equilibrium is unique and that action is aligned with Bonacich centrality. However, no equilibrium exists under strategic complements and large network effects. Agents' actions feed back into each other in an explosive way and diverge to infinity. This divergence is unrealistic in contexts where actions are naturally bounded. In their empirical implementation on peer effects and academic achievement, Calvó-Armengol et al. (2009) discuss this possibility:

"Let us bound the strategy space in such a game rather naturally by simply acknowledging the fact that students have a time constraint and allocate their time between leisure and school work. In that case, multiple equilibria will certainly emerge, which is a plausible outcome in the school setting.", Calvó-Armengol et al. (2009, p. 1254)

We disprove their conjecture for positive linear interactions. We extend Ballester et al. (2006)'s analysis to situations with large positive network effects and bounded actions. We show that uniqueness holds. We also show that equilibrium properties differ quite deeply for small and large interactions. Under small interactions, a more central agent always plays a higher action and a shock on one agent ends up affecting every other agent in connected networks. In contrast under large interactions, a more central agent may play a lower action. And an individual shock may not propagate throughout the network.

Two recent papers study non-linear best responses in network contexts. Hiller (2012) and Baetz (forthcoming) analyze network games with homogeneous concave best responses. They establish uniqueness by applying Kennan (2001)'s results. Both papers then look at network formation. In contrast, we consider fixed networks and heterogeneous best responses here. We also show that Kennan (2001)'s results apply. We provide an alternative approach, covering concave as well as important cases of non-concave best responses. We then investigate structural properties of the equilibria.

Our study also contributes to the literature on supermodular games. ${ }^{4}$ We provide one of the first crossovers between the theories of network games and of supermodular games. Galeotti et al. (2010) study network games under strategic complements when agents have incomplete information on the network. We analyze a game of complete information here. ${ }^{5}$ Belhaj and Deroïan (2010) study communication efforts under strategic complements and indirect network interactions, when agents play on specific symmetric networks. In contrast, we analyze direct network interactions and arbitrary networks. Our analysis notably applies to two important contexts: criminal activity and arms race. We combine classical results from the theory of supermodular games with network arguments to understand structural features of the equilibria.

The rest of the paper is organized as follows. We present the model and discuss applications in Section 2. We derive new sufficient conditions for uniqueness in Section 3. We study the relation between network position and action and comparative statics in Section 4. We conclude in Section 5.

\section{The model}

We consider the following network games of strategic complements. Society is composed of $n$ agents embedded in a fixed network, represented by an $n \times n$ matrix $\mathbf{G}$. The $(i, j)$ th entry $g_{i j}$ is a non-negative real number representing the link between $i$ and $j$. We consider an arbitrary weighted network. Formally $g_{i j} \in \mathbb{R}_{+}$and, by convention, $g_{i i}=0$. Agent $i$ is directly affected by agent $j$ if $g_{i j}>0$ and $g_{i j}$ then measures the strength of their relationship. We do not impose symmetry so $g_{i j}$ could differ from $g_{j i}$.

Agents choose actions $x_{i} \in\left[0, b_{i}\right]$ and play a game with best response

$$
f_{i}\left(\mathbf{x}_{-i}\right)=\min \left(a_{i}+\varphi_{i}\left(\sum_{j=1}^{n} g_{i j} x_{j}\right), b_{i}\right)
$$

where $b_{i}>0$ denotes the upper bound on $i$ 's action; $a_{i}$ denotes $i$ 's optimal action absent social interactions with $0<a_{i}<b_{i}$; and $\varphi_{i}$ is a continuously differentiable function from $\mathbb{R}_{+}$into $\mathbb{R}_{+}$such that $\varphi_{i}(0)=0$ and $\varphi_{i}^{\prime}>0$. The function $\varphi_{i}$ captures

\footnotetext{
3 See, for instance, Ballester et al. (2006), Belhaj and Deroïan (2013), Bramoullé and Kranton (2007), Bramoullé et al. (2014), Calvó-Armengol et al. (2009).

4 See, for instance, Milgrom and Roberts (1990) and Vives (1990).

5 However, we observe that an agent only needs to know his neighbors' actions to be able to play a best response.
} 
the impact of neighbors' actions below the upper bound. A Nash equilibrium of the game is a profile of actions $\mathbf{x}$ such that $\forall i, x_{i}=f_{i}\left(\mathbf{x}_{-i}\right)$.

Our model has three distinguishing features with respect to the recent literature on network games. First, we focus on strategic complements. An agent's best response is weakly increasing in others' actions. ${ }^{6}$ Second, actions are bounded from above by exogenously given upper bounds. As shown below, this helps guarantee equilibrium existence. Third, interactions need not be linear or concave. The functions $\varphi_{i}$ could be locally or globally convex.

We can apply classical results of the theory of supermodular games to study games with best responses (1). To see why, consider the game $\Gamma$ with strategies $x_{i} \in\left[0, b_{i}\right]$ and payoffs

$$
u_{i}\left(x_{i}, \mathbf{x}_{-i}\right)=a_{i} x_{i}-\frac{1}{2} x_{i}^{2}+x_{i} \varphi\left(\sum_{j=1}^{n} g_{i j} x_{j}\right)
$$

Note that $\left[0, b_{i}\right]$ is a complete lattice and $\partial^{2} u_{i} / \partial x_{i} \partial x_{j}=g_{i j} \varphi^{\prime}\left(\sum_{j=1}^{n} g_{i j} x_{j}\right) \geq 0, \forall i \neq j$. Therefore, $\Gamma$ is supermodular (Milgrom and Roberts, 1990). In particular, $\Gamma$ always has a smallest and a largest Nash equilibrium. And since Nash equilibria only depend on the best responses, this property holds for any game with best responses (1). To sum up,

Corollary 1. (See Milgrom and Roberts, 1990.) Any game with best response (1) has a smallest and a largest Nash equilibrium.

Such positive network interactions may emerge in a variety of contexts. We next describe two examples in some detail: crime and arms race. Researchers have documented the powerful role played by social interactions in determining criminal activity (Glaeser et al., 1996; Bayer et al., 2009). Building on Calvó-Armengol and Zenou (2004), Liu et al. (2014) model choices of criminal efforts in a delinquent network as Nash equilibria of a game with payoff functions

$$
v_{i}\left(x_{i}, \mathbf{x}_{-i}\right)=a_{i} x_{i}-\frac{1}{2} x_{i}^{2}+\delta \sum_{i} g_{i j} x_{i} x_{j}
$$

where effort is assumed to be non-negative, $x_{i} \in \mathbb{R}_{+}$. Here $a_{i}>0$ includes individual determinants of criminal activity while $\delta \sum_{i} g_{i j} x_{i} x_{j}$ captures the influence of peers' behaviors. In this case, individual best response is simply linear:

$$
f_{i}\left(\mathbf{x}_{-i}\right)=a_{i}+\delta \sum_{j=1}^{n} g_{i j} x_{j}
$$

Linear network games have been analyzed in Ballester et al. (2006) and Ballester and Calvó-Armengol (2010). Observe that if $\mathbf{x}$ is an equilibrium, we have $\mathbf{x}=\mathbf{a}+\delta \mathbf{G} \mathbf{x}$ and hence, through repeated substitutions,

$$
\mathbf{x}=\mathbf{a}+\delta \mathbf{G} \mathbf{a}+\delta^{2} \mathbf{G}^{2} \mathbf{a}+\cdots+\delta^{t} \mathbf{G}^{t} \mathbf{a}+\delta^{t+1} \mathbf{G}^{t+1} \mathbf{x}
$$

for any $t \in \mathbb{N}$. Denote by $\lambda_{\max }(\mathbf{G})$ the largest eigenvalue of matrix $\mathbf{G}$. There are two cases. If $\delta \lambda_{\max }(\mathbf{G})<1$, the previous series converges and there is a unique equilibrium given by $\mathbf{x}=(\mathbf{I}-\delta \mathbf{G})^{-1} \mathbf{a}$. Under homogeneity $\left(\forall i, a_{i}=a\right)$, actions are aligned with Bonacich centralities in the network. ${ }^{7}$ A more central agent always plays a higher action. In contrast if $\delta \lambda$ max $(\mathbf{G}) \geq 1$, the previous series diverges and there is no equilibrium. Faced with this existence issue, researchers have, so far, typically imposed the assumption that $\delta \lambda_{\max }(\mathbf{G})<1$. This is not very satisfactory. It means restricting attention to relatively small network effects. And this inequality may not hold empirically (Calvó-Armengol et al., 2009).

The assumption that action is unbounded is unrealistic in most empirical contexts, however. With crime, presumably, $x_{i}$ reaches some natural upper bound $b_{i}$ for individuals who have become full-time criminals and are not engaged in legal activities any longer. If we keep the same payoffs as in Liu et al. (2014) and introduce upper bounds $b_{i}$, the best response becomes a censored version of (3):

$$
f_{i}\left(\mathbf{x}_{-i}\right)=\min \left(a_{i}+\delta \sum_{j=1}^{n} g_{i j} x_{j}, b_{i}\right)
$$

Best response (4) is a particular case of (1) with $\varphi_{i}(x)=\delta x$, and hence an equilibrium now exists for any $\delta$. In addition, the assumption that the marginal impact of peers' actions on own marginal utility is constant and the same for everyone is quite specific. In reality, this marginal impact may well depend on the individual and on the level of peers' actions, which would lead to payoffs (2) and best responses (1). For instance, an individual may be more susceptible to criminal influence once his friends are really committed to criminal activity $\left(\varphi_{i}\right.$ convex) even though such susceptibility may then taper off as friends get close to full-time crime ( $\varphi_{i}$ concave).

\footnotetext{
6 See Bramoullé and Kranton (2007) and Bramoullé et al. (2014) for an analysis of network games with linear best responses and strategic substitutes.

7 The profile of Bonacich centralities is defined as $\mathbf{c}=(\mathbf{I}-\delta \mathbf{G}) \mathbf{G 1}$ (Bonacich, 1987), which yields $\mathbf{x}=a(\mathbf{1}+\delta \mathbf{c})$.
} 
Arms race provides another context where network effects matter. Milgrom and Roberts (1990) model arms race between two countries as the outcome of a game with strategies $x_{i} \in[0, b]$ and payoffs $\pi_{i}\left(x_{i}, x_{-i}\right)=B\left(x_{i}-x_{-i}\right)-C\left(x_{i}\right)$. To extend this model to many countries, relationships between countries have to be taken into account. Military alliances and historical partnerships likely shape up the interaction in arms' expenditures. A country probably worries more about the increase in the armament of a potential foe than of a long-time partner and ally. Thus, one way to extend this model to $n$ countries is to assume that

$$
\pi_{i}\left(x_{i}, x_{-i}\right)=B\left(x_{i}-\sum_{j=1}^{n} g_{i j} x_{j}\right)-C\left(x_{i}\right)
$$

where $g_{i j}=1$ if country $j$ is a potential foe of country $i$. Here, the network $\mathbf{G}$ represents antagonistic relationships. In what follows, we assume that $B$ and $C$ are twice continuously differentiable, $B$ is strictly increasing and strictly concave, $C$ is strictly increasing and strictly convex, $B^{\prime}(0)>C^{\prime}(\infty)$ and $B^{\prime}(\infty)<C^{\prime}(0)$.

Denote by $a$ the unique value such that $B^{\prime}(a)=C^{\prime}(a)$ where $a$ represents optimal armament in isolation. Denote by $I(x)=x$ the identity function and introduce $\varphi=\left(I-\left(B^{\prime}\right)^{-1} \circ C^{\prime}\right)^{-1}-a$ the function such that: ${ }^{8}$

$$
\forall x, \quad B^{\prime}(\varphi(x)+a-x)=C^{\prime}(\varphi(x)+a)
$$

Then, observe that the best response of the arms race game between $n$ countries is given by $f_{i}\left(\mathbf{x}_{-i}\right)=\min (a+$ $\left.\varphi\left(\sum_{j=1}^{n} g_{i j} x_{j}\right), b\right)$. A country tends to increase his armament when his potential opponents increase theirs, and the impact of others' armament levels depends on the benefits and costs. In addition if benefits $B_{i}$ and costs $C_{i}$ are heterogeneous, we would have heterogeneous armaments in isolation $a_{i}$ and impacts of enemies' arms $\varphi_{i}$ and hence a particular case of best response (1).

Our main objective in the remainder of the paper is to analyze the Nash equilibria of games with best responses (1). We investigate three issues in particular. When is there a unique equilibrium? How does the position in the network of interactions affect equilibrium action? And how do Nash equilibria depend on the parameters of the game?

\section{Uniqueness}

In this section, we identify a new class of games with strategic complements which have a unique equilibrium. We derive formal conditions under which the iterated best response is contracting over ordered profiles lying between the extremal equilibria. This localized contraction leads to uniqueness in this subset and, since it contains all equilibria, to global uniqueness. This may happen even with large network effects and when the best response is non-contracting over large portions of the original strategy space.

We first derive our main technical result. We provide a sufficient condition for uniqueness involving the smallest and the largest equilibrium. We then build on this condition to prove uniqueness under various assumptions on the game's exogenous parameters. Let $\mathbf{X}$ and $\mathbf{X}$ denote the smallest and largest equilibrium and denote by $I=\left\{i: x_{i}<b_{i}\right\}$ the set of agents who play an interior action in the smallest equilibrium.

Lemma 1. If $\forall i \in I, a_{i}+\varphi_{i}\left(\sum_{j=1}^{n} g_{i j} x_{j}\right)>K_{i}\left(\sum_{j=1}^{n} g_{i j} x_{j}\right)$ where $K_{i}=\sup \varphi_{i}^{\prime}$ over $\left[\sum_{j=1}^{n} g_{i j} x_{j}, \sum_{j=1}^{n} g_{i j} X_{j}\right]$, then the game has a unique equilibrium.

Proof. We prove this result in four steps.

1. Since $\mathbf{x}$ is an equilibrium, $\forall i, x_{i}=f_{i}\left(\mathbf{x}_{-i}\right)$ and hence,

$$
\forall i \in I, \quad x_{i}=a_{i}+\varphi_{i}\left(\sum_{j=1}^{n} g_{i j} x_{j}\right)
$$

Subtract $K_{i}\left(\sum_{j=1}^{n} g_{i j} x_{j}\right)$ from both sides of the equality and apply the Lemma's conditions:

$$
\forall i \in I, \quad x_{i}-K_{i} \sum_{j=1}^{n} g_{i j} x_{j}>0
$$

2. Denote by $\mathbf{H}$ the matrix defined over $I$ such that $\forall i, j \in I, h_{i j}=K_{i} g_{i j}$. Introduce $z_{i}=x_{i}-K_{i} \sum_{j=1}^{n} g_{i j} x_{j}, \forall i \in I$. Denote by $\mathbf{x}_{I}$ the restriction of profile $\mathbf{x}$ to $I$. Through successive substitutions, we can write:

$$
\mathbf{x}_{I}=\mathbf{z}+\mathbf{H} \mathbf{x}_{I}=\mathbf{z}+\mathbf{H z}+\mathbf{H}^{2} \mathbf{x}_{I}=\cdots=\sum_{s=0}^{t} \mathbf{H}^{s} \mathbf{z}+\mathbf{H}^{t+1} \mathbf{x}_{I}
$$

for any integer $t$. Since $\forall i \in I, z_{i}>0$ and $x_{i}>0$, this is only possible if $\mathbf{H}^{t}$ converges to $\mathbf{0}$ as $t$ tends to infinity.

8 The function $\varphi$ is well-defined since $I-\left(B^{\prime}\right)^{-1} \circ C^{\prime}$ is strictly increasing. 
3. Let $S=\left\{\mathbf{y} \in \mathbb{R}_{+}^{n}: \mathbf{x} \leq \mathbf{y} \leq \mathbf{X}\right\}$ be the set of action profiles lying between the smallest and largest Nash equilibrium. Observe first that $\mathbf{f}(S) \subset S$. Since $\mathbf{f}$ is weakly increasing and $\mathbf{x}$ and $\mathbf{X}$ are equilibria, the best response of any profile in $S$ lies in $S$. Next, for any $\mathbf{y}, \mathbf{y}^{\prime} \in S$ such that $\mathbf{y} \leq \mathbf{y}^{\prime}$, we have:

$$
\mathbf{0} \leq \mathbf{f}\left(\mathbf{y}^{\prime}\right)-\mathbf{f}(\mathbf{y}) \leq \varphi\left(\mathbf{y}^{\prime}\right)-\varphi(\mathbf{y}) \leq \mathbf{H}\left(\mathbf{y}^{\prime}-\mathbf{y}\right)
$$

where the first inequality follows from the monotonicity of the best response; the second inequality from the examination of possible cases ${ }^{9}$ and the third inequality from the mean value theorem and the definition of $K_{i}$ and $\mathbf{H}$.

4. Apply the previous inequality to $\mathbf{f}(\mathbf{y})$ and $\mathbf{f}\left(\mathbf{y}^{\prime}\right): \mathbf{0} \leq \mathbf{f}^{2}\left(\mathbf{y}^{\prime}\right)-\mathbf{f}^{2}(\mathbf{y}) \leq \mathbf{H}\left(\mathbf{f}\left(\mathbf{y}^{\prime}\right)-\mathbf{f}(\mathbf{y})\right) \leq \mathbf{H}^{2}\left(\mathbf{y}^{\prime}-\mathbf{y}\right)$ since $\mathbf{H}$ has non-negative entries. By induction, $\mathbf{0} \leq \mathbf{f}^{t}\left(\mathbf{y}^{\prime}\right)-\mathbf{f}^{t}(\mathbf{y}) \leq \mathbf{H}^{t}\left(\mathbf{y}^{\prime}-\mathbf{y}\right)$ for any $t \geq 1$. Since $\mathbf{H}^{t}$ converges to 0 , there exists $C<1$ such that $\left\|\mathbf{f}^{t}\left(\mathbf{y}^{\prime}\right)-\mathbf{f}^{t}(\mathbf{y})\right\| \leq C\left\|\mathbf{y}^{\prime}-\mathbf{y}\right\|$ for $t$ high enough. Apply to $\mathbf{y}=\mathbf{x}$ and $\mathbf{y}^{\prime}=\mathbf{X}$. Since $\mathbf{f}^{t}(\mathbf{x})=\mathbf{x}$ and $\mathbf{f}^{t}(\mathbf{X})=\mathbf{X},\|\mathbf{X}-\mathbf{x}\| \leq C\|\mathbf{X}-\mathbf{x}\|$ and $\mathbf{X}-\mathbf{x}=\mathbf{0}$. The game has a unique Nash equilibrium.

We now show how to apply Lemma 1 to prove uniqueness for various assumptions on the games' exogenous parameters. Consider linear best responses first: $\varphi_{i}(u)=\delta_{i} u$. In that case, observe that $K_{i}=\delta_{i}$ and $a_{i}+\varphi_{i}\left(\sum_{j=1}^{n} g_{i j} x_{j}\right)=$ $a_{i}+\delta_{i}\left(\sum_{j=1}^{n} g_{i j} x_{j}\right)>\delta_{i}\left(\sum_{j=1}^{n} g_{i j} x_{j}\right)$ since $a_{i}>0$. Thus, the conditions of Lemma 1 always hold when the $\varphi_{i}$ 's are linear. More generally, we show next that these conditions necessarily hold when the $\varphi_{i}$ 's are concave. Under concavity, Kennan (2001)'s classical results also apply here. Kennan (2001)'s analysis and ours are based on very different premises, however. Our approach thus provides an alternative way to show uniqueness under concavity.

Proposition 1. Suppose that $\forall i, \varphi_{i}$ is concave. Then, the game has a unique Nash equilibrium.

Proof. We derive two alternative proofs of this result. We show that Lemma 1 and Theorem 1 of Kennan (2001) both apply here.

1. Since $\varphi_{i}$ is concave and $\varphi_{i}(0)=0, \forall u \geq 0, \varphi_{i}(u) \geq u \varphi_{i}^{\prime}(u)$. This implies that:

$$
a_{i}+\varphi_{i}\left(\sum_{j=1}^{n} g_{i j} x_{j}\right)>\varphi_{i}\left(\sum_{j=1}^{n} g_{i j} x_{j}\right) \geq \varphi_{i}^{\prime}\left(\sum_{j=1}^{n} g_{i j} x_{j}\right)\left(\sum_{j=1}^{n} g_{i j} x_{j}\right)=K_{i}\left(\sum_{j=1}^{n} g_{i j} x_{j}\right)
$$

where the equality comes from the fact that $\varphi_{i}^{\prime}$ is weakly decreasing. Therefore, the condition of Lemma 1 holds and uniqueness follows.

2. Introduce $\mathbf{h}(\mathbf{x})=\mathbf{f}(\mathbf{x})-\mathbf{x}$. Since $\mathbf{f}$ is weakly increasing, $\mathbf{h}$ is "quasi-increasing". ${ }^{10}$ We next show that $\mathbf{h}$ is "strictly radially quasiconcave". Consider $\mathbf{x}$ such that $\mathbf{h}(\mathbf{x})=\mathbf{0}$ and $\lambda \in] 0,1\left[\right.$. We need to show that $\forall i, h_{i}(\lambda \mathbf{x})>0$. There are two cases. (1) If $x_{i}=a_{i}+\varphi_{i}\left(\sum_{j} g_{i j} x_{j}\right)$, then $h_{i}(\lambda \mathbf{x})=(1-\lambda) a_{i}+\varphi_{i}\left(\lambda \sum_{j} g_{i j} x_{j}\right)-\lambda \varphi_{i}\left(\sum_{j} g_{i j} x_{j}\right)$. Since $\varphi_{i}$ is concave and $\varphi_{i}(0)=$ $0, \varphi_{i}(\lambda u) \geq \lambda \varphi_{i}(u) \forall u>0$ and $h_{i}(\lambda \mathbf{x}) \geq(1-\lambda) a_{i}>0$. (2). If $x_{i}=b_{i}$, then $h_{i}(\lambda \mathbf{x})=f_{i}\left(\lambda \mathbf{x}_{-i}\right)-\lambda b_{i}$. If $f_{i}\left(\lambda \mathbf{x}_{-i}\right)=b_{i}$, then $h_{i}(\lambda \mathbf{x})=(1-\lambda) b_{i}>0$. Otherwise, $f_{i}\left(\lambda \mathbf{x}_{-i}\right)=a_{i}+\varphi_{i}\left(\lambda \sum_{j} g_{i j} x_{j}\right) \geq(1-\lambda) a_{i}+\lambda\left(a_{i}+\varphi_{i}\left(\sum_{j} g_{i j} x_{j}\right)\right) \geq(1-\lambda) a_{i}+\lambda b_{i}$ and $h_{i}(\lambda \mathbf{x}) \geq(1-\lambda) a_{i}>0$. Therefore, Theorem 1 in Kennan (2001) applies.

We consider convex functions next. We can see that strong convexity in the best responses easily leads to multiple equilibria. For uniqueness to hold under convexity, the extent of the curvature must be somehow bounded. This is precisely what we obtain when applying Lemma 1 to convex functions. Define $B_{i}=\sum_{j} g_{i j} b_{j}$ as the largest possible level of neighbors' actions, from agent $i$ 's point of view.

Proposition 2. Suppose that $\forall i, \varphi_{i}$ is convex. If $\forall i, a_{i}+\varphi_{i}\left(B_{i}\right)>\varphi_{i}^{\prime}\left(B_{i}\right) B_{i}$, then the game has a unique Nash equilibrium.

Proof. Since $\varphi_{i}$ is convex, $\varphi_{i}^{\prime}$ is weakly increasing and $K_{i}=\varphi_{i}^{\prime}\left(\sum_{j=1}^{n} g_{i j} X_{j}\right) \leq \varphi_{i}^{\prime}\left(B_{i}\right)$. Consider the auxiliary function $\psi_{i}(u)=$ $a_{i}+\varphi_{i}(u)-\varphi_{i}^{\prime}\left(B_{i}\right) u$. We have: $\psi_{i}^{\prime}(u)=\varphi_{i}^{\prime}(u)-\varphi_{i}^{\prime}\left(B_{i}\right) \leq 0$ if $u \leq B_{i}$. Therefore, $\psi_{i}$ is weakly decreasing over $\left[0, B_{i}\right]$ and $\psi_{i}\left(\sum_{j=1}^{n} g_{i j} x_{j}\right) \geq \psi_{i}\left(B_{i}\right)$. Note that $\psi_{i}\left(B_{i}\right)>0$ amounts precisely to the condition stated in the Proposition. This means that $a_{i}+\varphi_{i}\left(\sum_{j=1}^{n} g_{i j} x_{j}\right)>\varphi_{i}^{\prime}\left(B_{i}\right)\left(\sum_{j=1}^{n} g_{i j} x_{j}\right) \geq K_{i}\left(\sum_{j=1}^{n} g_{i j} x_{j}\right)$ and Lemma 1's condition holds.

To illustrate this result, consider functions with constant elasticity: $\varphi_{i}(u)=\delta_{i} u^{\eta_{i}}$ with $\eta_{i}>1$. Proposition 2's sufficient condition becomes:

$$
a_{i}>\delta_{i}\left(\eta_{i}-1\right) B_{i}^{\eta_{i}}
$$

which is more easily satisfied when $a_{i}$ is large, $\delta_{i}$ is small and, if $B_{i} \geq 1$, when $\eta_{i}$ is closer to 1 .

\footnotetext{
${ }^{9}$ If $f_{i}\left(\mathbf{y}_{-i}\right)=b_{i}$, then $f_{i}\left(\mathbf{y}_{-i}^{\prime}\right)=b_{i}$. If $f_{i}\left(\mathbf{y}_{-i}\right)<b_{i}$, then $f_{i}\left(\mathbf{y}_{-i}^{\prime}\right)-f_{i}\left(\mathbf{y}_{-i}\right)=f_{i}\left(\mathbf{y}_{-i}^{\prime}\right)-\left(a_{i}+\varphi_{i}\left(\sum_{j} g_{i j} y_{j}\right)\right) \leq \varphi_{i}\left(\sum_{j} g_{i j} y_{j}^{\prime}\right)-\varphi_{i}\left(\sum_{j} g_{i j} y_{j}\right)$.

10 We refer to Kennan (2001) for details and formal definitions.
} 
Finally, we derive a sufficient uniqueness condition for functions with arbitrary curvature. Define $A_{i}=\sum_{j} g_{i j} a_{j}$ as the smallest possible level of neighbors' actions.

Proposition 3. If $\forall i, a_{i}+\varphi_{i}\left(B_{i}\right)>K_{i}^{\prime} B_{i}$ with $K_{i}^{\prime}=\sup \varphi_{i}^{\prime}$ over $\left[A_{i}, B_{i}\right]$, then the game has a unique equilibrium.

Proof. Let $u \in\left[A_{i}, B_{i}\right]$. By assumption, $a_{i}+\varphi_{i}\left(B_{i}\right)>K_{i}^{\prime} B_{i}$. Subtracting $\varphi_{i}(u)$ yields $\varphi_{i}\left(B_{i}\right)-\varphi_{i}(u)>K_{i}^{\prime} B_{i}-a_{i}-\varphi_{i}(u)$. By the mean value theorem, $\varphi_{i}\left(B_{i}\right)-\varphi_{i}(u) \leq K_{i}^{\prime}\left(B_{i}-u\right)$. Combining both inequalities leads to

$$
a_{i}+\varphi_{i}(u)>K_{i}^{\prime} u
$$

Since $K_{i}^{\prime} \geq K_{i}$, applying in $u=\sum_{j=1}^{n} g_{i j} x_{j}$ leads to

$$
a_{i}+\varphi_{i}\left(\sum_{j=1}^{n} g_{i j} x_{j}\right)>K_{i}\left(\sum_{j=1}^{n} g_{i j} x_{j}\right)
$$

and Lemma 1 applies.

Therefore, uniqueness may prevail even in the presence of convexities and large network effects. When positive feedbacks are initially explosive, some agents will quickly reach their upper bounds. This tends to dampen the interaction and this dampening may be sufficiently strong to yield uniqueness. Our results clarify the circumstances under which the best response satisfies a contracting property between the extremal equilibria. Such localized contraction always holds for linear and concave best responses. It may also hold under convex best responses (below the upper bound) when the convexity is not too strong. While the uniqueness results for concave best responses are not new, they had not been connected to localized contraction before and the results for convex and arbitrary functions are new.

Even though simple analytical expressions for equilibrium actions are generally out of reach, they can be computed easily with the help of classical algorithms. In particular, we know that in these games repeated myopic best responses converge rapidly and monotonically towards the smallest equilibrium when starting from the smallest profile $\left(\forall i, x_{i}=0\right)$ and towards the largest equilibrium when starting from the largest profile $\left(\forall i, x_{i}=b_{i}\right)$ (Vives, 1990; Echenique, 2007). Thus, both dynamic processes converge towards the equilibrium under uniqueness and we make use of these algorithms in our examples below.

In the next section, we analyze the properties of Nash equilibria in some detail. We first look at how positions in the network affect actions. We then focus on comparative statics. Our results apply to any game with best responses (1). Under multiplicity, they apply to extremal equilibria and in one instance, to all equilibria. Under uniqueness, they help characterize the equilibrium's structural properties.

\section{Equilibrium properties}

\subsection{Network position and action}

In this section, we study how an agent's position in the network affects his action in equilibrium. To focus on the effect of the structure, we assume throughout the section that agents can only differ in their network positions and that links are binary. Formally, $\forall i, a_{i}=a, b_{i}=b, \varphi_{i}=\varphi$ and $\forall i, j, g_{i j} \in\{0,1\}$. Best responses are then given by:

$$
f_{i}\left(\mathbf{x}_{-i}\right)=\min \left(a+\varphi\left(\sum_{j=1}^{n} g_{i j} x_{j}\right), b\right)
$$

We establish, first, that the upper bound has a strong impact on the relationship between position and action. Consider linear best responses: $\varphi(u)=\delta u$. With no upper bound and small network effects, equilibrium actions are aligned with the Bonacich centralities of the agents in the network (Ballester et al., 2006). We show now that this alignment may not hold in the presence of an upper bound.

Consider the network depicted in Fig. 1. It has eight nodes and two cliques: one composed of agents 1 to 4 and the triangle 6-7-8. ${ }^{11}$ In addition, agent 5 in the middle is connected to agents 4 and 6 . When $\delta=0.3$ and actions are unbounded, the equilibrium is such that $x_{5} \approx 7.9>x_{6} \approx 5.7 .{ }^{12}$ Even though agent 6 has one more neighbor than agent 5 , his neighbors are not very central. In contrast, agent 5 is connected to agent 4 who is the most central agent in the graph. When $\delta$ is not too low, the effect of indirect paths dominate; agent 5 is more central than agent 6 and plays a higher action. Suppose next that actions are bounded from above by $L=5$. The equilibrium is now such that $x_{5} \approx 3.7<x_{6} \approx 4.0{ }^{13}$ Agents $1-4$ reach

\footnotetext{
11 A clique is a completely connected subgraph.

12 The whole equilibrium profile is $\mathbf{x} \approx(15.46,15.46,15.46,17.28,7.89,5.69,3.87,3.87)$.

13 The whole equilibrium profile is now $\mathbf{x} \approx(5,5,5,5,3.70,3.99,3.14,3.14)$.
} 


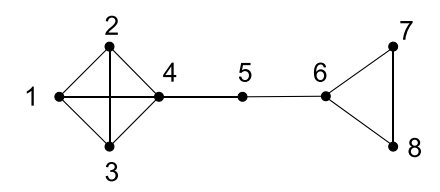

Fig. 1. A network for which action and centrality may not be aligned.

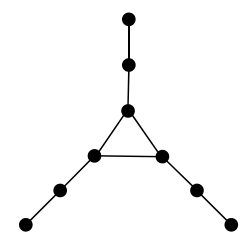

Fig. 2. An example of hierarchical community.

the upper bound and this reduces the action premium that agent 5 gets from his link with agent 4 . Agent 6 , who is less central, now plays a higher action.

We next identify specific situations leading to a clear-cut relationship between position and action. Say that agent $i$ has fewer neighbors than agent $j$ in the sense of set inclusion if $\forall k \neq i, j, g_{i k} \leq g_{j k}$. Agents $i$ and $j$ could be connected. For instance in the graph depicted in Fig. 1, agents 7 and 8 have fewer neighbors than agent 6 and agents 1, 2 and 3 have fewer neighbors than agent 4 in the sense of set inclusion. Agents $i$ and $j$ could also be disconnected. For instance, if the link between 7 and 8 is removed in Fig. 1, agents 7 and agents 8 now have fewer neighbors than agent 5 in the sense of set inclusion. In these situations, the actions of the two agents can always be ordered.

Proposition 4. Let $\mathbf{x}$ be any Nash equilibrium of a game with best responses (5). If agent $i$ has fewer neighbors than agent $j$ in the sense of set inclusion, then $x_{i} \leq x_{j}$.

Proof. Let $\mathbf{x}$ be a Nash equilibrium. Suppose first that $g_{i j}=0$. Then, $\sum_{k} g_{i k} x_{k} \leq \sum_{k} g_{j k} x_{k}$. Therefore, $\varphi\left(\sum_{k} g_{i k} x_{k}\right) \leq$ $\varphi\left(\sum_{k} g_{j k} x_{k}\right)$ and $\min \left(a+\varphi\left(\sum_{k} g_{i k} x_{k}\right), b\right) \leq \min \left(a+\varphi\left(\sum_{k} g_{j k} x_{k}\right), b\right)$. Since $\mathbf{x}$ is an equilibrium, this means that $x_{i} \leq x_{j}$. Suppose next that $g_{i j}=1$. Suppose that $x_{i}>x_{j}$. This implies that $\sum_{k} g_{i k} x_{k}>\sum_{k} g_{j k} x_{k}$ and $x_{j}+\sum_{k \neq i, j} g_{i k} x_{k}>x_{i}+\sum_{k \neq i, j} g_{j k} x_{k}$ Since $\sum_{k \neq i, j} g_{i k} x_{k} \leq \sum_{k \neq i, j} g_{j k} x_{k}$, then $x_{j}>x_{i}$ which establishes a contradiction. Therefore, $x_{i} \leq x_{j}$.

We emphasize that Proposition 4 holds for any strictly increasing function $\varphi$. This result therefore uncovers a robust property of network games with strategic complements. A nice implication is that it leads to a full ranking of the actions for nested split graphs. A graph is nested split if agents can be ordered so that $g_{k l}=1 \Rightarrow g_{i j}=1$ whenever $i \leq k$ and $j \leq l$. These graphs emerge, for instance, as outcomes of centrality-based models of network formation (König et al., 2014) and as solutions of network design problems (Belhaj et al., 2013). On nested split graphs, if agent $i$ has a lower degree than agent $j$ then she has fewer neighbors in the sense of set inclusion. By Proposition 4, we then have $x_{i} \leq x_{j}$. If agent $i$ has the same degree as agent $j$, there are two possibilities. Either they are unconnected and they have the same neighbors: $\forall k, g_{i k}=g_{j k}$. Or they are connected and $\forall k \neq i, j, g_{i k}=g_{j k}$. In either case, Proposition 4 implies that $x_{i}=x_{j}$.

Corollary 2. On nested split graphs, action is an increasing function of degree in any Nash equilibrium of a game with best response (5).

Another feature that may help connect position and action is geometric symmetry. In particular, the analysis of Belhaj and Deroïan (2010) applies here. They study supermodular network games played on the line and on more general "hierarchical communities". These networks are defined by the following three features. (1) They are structured around a geometric center. (2) Degrees decreases weakly as nodes get further away from the center. (3) All agents at a given distance from the center have symmetric positions. An example of such graphs is given in Fig. 2; we refer to Belhaj and Deroïan (2010) for more details and formal definitions. In these structures, even though neighborhoods are not nested centrality is unambiguous. An agent is more central when her distance to the center is lower. Their main result states that in these graphs, action is aligned with centrality in the smallest and largest Nash equilibrium. Their analysis directly applies to our setup.

Corollary 3. (See Belhaj and Deroïan, 2010.) On the line and on more general hierarchical communities, action is aligned with centrality in the extremal Nash equilibria. 


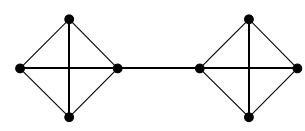

Fig. 3. Two communities connected by a bridge.

\subsection{Comparative statics and broken interdependence}

In this section, we study comparative statics of games with best response (1). We look, in particular, at how a shock propagates in the network. We first derive weak monotone comparative statics by applying classical results from the theory of supermodular games.

Corollary $\mathbf{4}$ (Weak monotone comparative statics). The action of any agent is weakly increasing in $\mathbf{a}$, $\mathbf{b}$ and $\mathbf{G}$ in the smallest and in the largest Nash equilibrium.

Proof. Compute payoff cross-derivatives in the game $\Gamma: \partial^{2} u_{i} / \partial x_{i} \partial a_{i}=1 ; \partial^{2} u_{i} / \partial x_{i} \partial a_{j}=0$ if $j \neq i$; $\partial^{2} u_{i} / \partial x_{i} \partial g_{i j}=$ $x_{j} \varphi^{\prime}\left(\sum_{k} g_{i k} x_{k}\right) ; \partial^{2} u_{i} / \partial x_{i} \partial g_{k l}=0$ if $k l \neq i j$. All these derivatives are greater than or equal to zero. By Theorem 6 in Milgrom and Roberts (1990), action $x_{i}$ in the extremal equilibria is then weakly increasing in all these parameters for any $i$. Next, define the sequence $\mathbf{y}^{n}(\mathbf{b})$ by $\mathbf{y}^{0}(\mathbf{b})=\mathbf{0}$ and $\mathbf{y}^{n+1}(\mathbf{b})=\mathbf{f}\left(\mathbf{y}^{n}(\mathbf{b}), \mathbf{b}\right)$. By induction, we see that $\mathbf{b} \leq \mathbf{b}^{\prime} \Rightarrow \mathbf{y}^{n}(\mathbf{b}) \leq \mathbf{y}^{n}\left(\mathbf{b}^{\prime}\right)$. Since $\mathbf{y}^{n}$ (b) converges to $\mathbf{x}(\mathbf{b})$ as $n$ tends to infinity, this means that $\mathbf{x}(\mathbf{b}) \leq \mathbf{x}\left(\mathbf{b}^{\prime}\right)$. A similar argument applies to the largest equilibrium.

Under strategic complements, direct and indirect network effects are fully aligned. Consider, for instance, the impact of an increase in $a_{i}$, the action of agent $i$ in isolation. The direct effect is to induce agent $i$ to increase his action. As a consequence, agent $i$ 's neighbors will also tend to increase their actions. In turn, their neighbors tend to increase their own actions. The impact of the original shock propagates throughout the network and all the direct and indirect effects are greater than or equal to zero. In the end, the action of every other agent increases weakly. This stands in sharp contrast to setups with strategic substitutes, where direct and indirect effects are generally not aligned and comparative statics can be quite complicated (Bramoullé et al., 2014).

Corollary 4 covers two different situations, however. An increase in $a_{i}$ could lead to an increase in $x_{j}$ or it could leave $x_{j}$ unaffected. Under linear best responses and small network effects, the second possibility is excluded. Interdependence is, in a sense, maximal. If $\varphi_{i}(u)=\delta u$ and $\delta$ small, then $\mathbf{x}=(\mathbf{I}-\delta \mathbf{G})^{-1} \mathbf{a}$ and, if $\mathbf{G}$ is connected, $\partial x_{i} / \partial a_{j}>0 \forall i, j$. The presence of upper bounds affects this property quite deeply. When an agent reaches his upper bound, he stops being a transmitter of influences in the network. Depending on how agents are placed, interdependence may then be broken and whole parts of the network may be unaffected by individual shocks.

Let us first illustrate how this can happen. Consider linear best responses under homogeneity, binary links and the graph depicted in Fig. 3. Society is composed of two communities of equal size. In each community, every agent is connected to every other agent. In addition, one agent in the first community is connected to one agent in the second. The two communities are bridged through a unique link. By Proposition 1, the equilibrium is unique. We can show that there exists two threshold levels $a_{1}^{*}$ and $a_{2}^{*}$ such that the following properties hold. If $a<a_{1}^{*}, \partial x_{i} / \partial a_{j}>0$ for any two agents $i$ and $j$ in the population. If $a_{1}^{*}<a<a_{2}^{*}$, then $x_{i}=b$ for both bridge agents; $\partial x_{i} / \partial a_{j}>0$ for any two non-bridge agents $i$ and $j$ in the same community while $\partial x_{i} / \partial a_{j}=0$ if $i$ lies in one community and $j$ lies in the other. Finally, if $a \geq a_{2}^{*}$, every agent plays the upper bound. When actions in isolation are small, the bridge plays a crucial role: it transmits shocks from one community to the other. As actions in isolation increase, bridge agents tend to reach the upper bound first because of their more central position. ${ }^{14}$ When this happens, they become unresponsive to further increases and this turns off the transmission channel.

We now analyze this issue more generally. Consider an extremal equilibrium. Denote by $P_{i}=\left\{j:\left(\frac{\partial x_{i}}{\partial a_{j}}\right)^{+}>0\right\}$ the set of agents $j$ who affect $i$ indirectly in the sense that an increase in $a_{j}$ leads to an increase in $x_{i} .{ }^{15}$ For instance, $P_{i}=N \backslash\{i\}$ under linear best responses, small network effects and a connected graph. In contrast, $P_{i}=\varnothing$ if $x_{i}=b_{i}$. This set can be simply characterized as follows. A path connecting $i$ to $j$ in $\mathbf{G}$ is a sequence of agents $i_{1}=i, i_{2}, \ldots, i_{l}=j$ such that $g_{i_{s} i_{s+1}}>0$, $\forall s \in\{1,2, \ldots, l-1\}$.

Proposition 5. In the smallest and largest equilibrium, agent $j$ affects agent $i$ indirectly if and only if there is a path from $i$ to $j$ in $\mathbf{G}$ in which all agents play an interior action.

\footnotetext{
14 Observe that a bridge agent in one community has more neighbors than other community members in the sense of set inclusion. By Proposition 4 , she always plays a weakly higher action.

15 The lowest and the highest equilibrium are continuous functions of a. The left- and right-derivatives of individual action $x_{i}$ with respect to $a_{j}$ may differ, however. We denote by $\left(\frac{\partial x_{i}}{\partial a_{j}}\right)^{+}$the right-derivative. Alternatively, we could consider the set of agents $j$ such that a decrease in $a_{j}$ leads to a decrease in $x_{i}$ and the analysis would carry over.
} 
Proof. Suppose that $x_{i}<b_{i}$ and denote by $I$ the set of agents playing an interior action. We have:

$$
\forall i \in I, x_{i}=a_{i}+\varphi_{i}\left(\sum_{k \in I} g_{i k} x_{k}+\sum_{k \notin I} g_{i k} b_{k}\right)
$$

The set $I$ does not change following an infinitesimal increase in $a_{j}$. Take the right-derivative with respect to $a_{j}$.

$$
\forall i \in I,\left(\frac{\partial x_{i}}{\partial a_{j}}\right)^{+}=\delta_{i j}+\sum_{k \in I} g_{i k}\left(\frac{\partial x_{k}}{\partial a_{j}}\right)^{+} \varphi_{i}^{\prime}\left(\sum_{k} g_{i k} x_{k}\right)
$$

where $\delta_{i j}=1$ if $i=j$ and 0 otherwise. Introduce the matrix $\mathbf{H}$ defined over $I$ such that $h_{i j}=g_{i j} \varphi_{i}^{\prime}\left(\sum_{k} g_{i k} x_{k}\right), \mathbf{e}^{j}$ the profile such that $e_{k}^{j}=\delta_{j k}$. In matrix notations, we have:

$$
\left(\frac{\partial \mathbf{x}_{I}}{\partial a_{j}}\right)^{+}=\mathbf{e}^{j}+\mathbf{H}\left(\frac{\partial \mathbf{x}_{I}}{\partial a_{j}}\right)^{+}
$$

By Corollary 4, $\left(\frac{\partial x_{i}}{\partial a_{j}}\right)^{+} \geq 0$. By an argument similar to the second step of the proof of Lemma 1, we must have $\lambda_{\max }(\mathbf{H})<1$. This means that $\mathbf{I}-\mathbf{H}$ is invertible and hence $\left(\frac{\partial \mathbf{x}_{l}}{\partial a_{j}}\right)^{+}=(\mathbf{I}-\mathbf{H})^{-1} \mathbf{e}^{j}$. This yields:

$$
\left(\frac{\partial x_{i}}{\partial a_{j}}\right)^{+}=\sum_{t=0}^{\infty}\left(\mathbf{H}^{t}\right)_{i j}
$$

Therefore, $j \in P_{i}$ iff there exists an integer $t$ such that $\left(\mathbf{H}^{t}\right)_{i j}>0$. This is equivalent to the fact that there is a path from $i$ to $j$ in $\mathbf{G}$ in which all agents play an interior action.

A direct implication of Proposition 5 is that the extent of interdependence necessarily decreases as actions in isolation increase.

Corollary 5. As actions in isolation a increase, the set of agents who affect $i$ indirectly, $P_{i}$, in the smallest and largest equilibrium shrinks monotonically towards the empty set.

Proof. By Corollary 4, more agents reach their upper bounds as a increases. By Proposition 5, $P_{i}$ shrinks. When $\mathbf{a}=\mathbf{b}$, $P_{i}=\varnothing \forall i$.

The way interdependence gets broken clearly depends on the shape of the network and on the existence and positions of bridges. When bridging agents are relatively more central within their own communities, we can expect interdependence to be reduced quite quickly because they will tend to reach their upper bounds first. However, there are also situations where agents who are better connected outside of their communities are less connected within. In these situations, interdependence may be more robust as bridging agents may tend to reach their upper bounds later.

\section{Conclusion}

In this paper, we analyze network games with positive but bounded actions and strategic complementarities. We derive new sufficient conditions for uniqueness and study the structural properties of the equilibria. We provide the first applications of a general principle of localized contraction and prove uniqueness for all concave as well as some non-concave best responses. We find that the upper bounds have a strong impact on the relationship between action and centrality. They may lead more central agents to play a lower action. Still, an agent who has more neighbors than another in the sense of set inclusion always plays a higher action. An increase in the game parameters necessarily leads to a weak increase in every agent's action. However, it may leave some agents unaffected. We characterize precisely when an agent is indirectly affected by a shock on another agent and uncover a general pattern of decreasing interdependence.

Our analysis could potentially be useful for empirical studies of peer effects in networks. A typical econometric model aimed at studying whether some outcome $x$ is subject to peer effects can be written:

$$
x_{i}=a_{i}+\delta \sum_{j} g_{i j} x_{j}+\varepsilon_{i}
$$


where $\delta$ is the main parameter of interest to be estimated and $\varepsilon_{i}$ is an error term. ${ }^{16}$ Most outcomes of interest, such as grades, criminal activity, or time spent exercising, are naturally bounded from above but existing empirical studies have neglected these bounds. This likely generates biases in existing estimates. To account for upper bounds properly in the estimation, a natural solution is to estimate a censored version of (6):

$$
x_{i}=\min \left(a_{i}+\delta \sum_{j} g_{i j} x_{j}+\varepsilon_{i}, b_{i}\right)
$$

This defines a system of simultaneous equations which is formally equivalent to the equilibrium conditions of a game with best response (1). Our results show that when outcomes are positive, this system has a unique solution. The empirical analysis thus does not suffer from the complications arising from equilibrium multiplicity (Tamer, 2003). Since the equilibrium $\mathbf{x}$ is a function of $\mathbf{a}, \delta, \mathbf{G}$ and $\varepsilon$ we can, in principle and given some assumptions on the error terms, compute the likelihood $L(\mathbf{x} \mid \mathbf{a}, \delta, \mathbf{G})$ and estimate $\delta$ through maximum likelihood in a straightforward manner. Moreover, our results show that this property carries over to heterogeneous and non-linear variants of model (6). Therefore, our analysis provides a stepping stone for empirical studies of peer effects in networks with continuous but bounded outcomes.

\section{References}

Baetz, Oliver, forthcoming. Social activity and network formation. Theoretical Econ.

Ballester, Coralio, Calvó-Armengol, Antoni, 2010. Interactions with hidden complementarities. Reg. Sci. Urban Econ. 40 (6), $397-406$.

Ballester, Coralio, Calvó-Armengol, Antoni, Zenou, Yves, 2006. Who's who in networks. Wanted: the key player. Econometrica 74 (5), 1403-1417.

Bayer, Patrick, Hjalmarsson, Randi, Pozen, David, 2009. Building criminal capital behind bars: peer effects in juvenile corrections. Quart. J. Econ. 124 (1), 105-147.

Belhaj, Mohamed, Bervoets, Sébastian, Deroïan, Frédéric, 2013. Network design under local complementarities. Working paper, Aix-Marseille University. Belhaj, Mohamed, Deroïan, Frédéric, 2010. Endogenous effort in communication networks under strategic complementarity. Int. J. Game Theory 39, $391-408$. Belhaj, Mohamed, Deroïan, Frédéric, 2013. Strategic interaction and aggregate incentives. J. Math. Econ. 49 (3), $183-188$.

Blume, Lawrence, Brock, William, Durlauf, Steven, Ioannides, Yannis, 2010. Identification of social interactions. In: Benhabib, J., Bisin, A., Jackson, M. (Eds.), Handbook of Social Economics. North Holland, Amsterdam.

Bonacich, P., 1987. Power and centrality: a family of measures. Amer. J. Sociology 92 (5), 1170-1182.

Bramoullé, Yann, Kranton, Rachel, 2007. Public goods in networks. J. Econ. Theory 135 (1), 478-494.

Bramoullé, Yann, Djebbari, Habiba, Fortin, Bernard, 2009. Identification of peer effects through social networks. J. Econometrics 150, 41-55.

Bramoullé, Yann, Kranton, Rachel, D’amours, Martin, 2014. Strategic interaction and networks. Amer. Econ. Rev. 104 (3), $898-930$.

Calvó-Armengol, Antoni, Zenou, Yves, 2004. Social networks and crime decisions: the role of social structure in facilitating delinquent behavior. Int. Econ. Rev. 45 (3), 939-958.

Calvó-Armengol, Antoni, Patacchini, Eleanora, Zenou, Yves, 2009. Peer effects and social networks in education. Rev. Econ. Stud. 76 (4), $1239-1267$.

De Giorgi, Giacomo, Pellizzari, Michele, Redaelli, Silvia, 2010. Identification of social interactions through partially overlapping peer groups. Amer. Econ. J. Appl. Econ. 2 (2), 241-275.

Echenique, Federico, 2007. Finding all equilibria in games of strategic complements. J. Econ. Theory 135, 514-532.

Galeotti, Andrea, Goyal, Sanjeev, Jackson, Matthew, Vega-Redondo, Fernando, Yariv, Leeat, 2010. Network games. Rev. Econ. Stud. 77 (1), $218-244$.

Glaeser, Edward, Sacerdote, Bruce, Scheinkman, José, 1996. Crime and social interactions. Quart. J. Econ. 111 (2), $507-548$.

Hiller, Timo, 2012. Peer effects in endogenous networks. Working paper, University of Bristol.

Kennan, John, 2001. Uniqueness of positive fixed points for increasing concave functions on $\mathbb{R}^{n}$ : an elementary result. Rev. Econ. Dynam. 4 (4), 893-899.

König, Michael, Tessone, Claudio, Zenou, Yves, 2014. Nestedness in networks: a theoretical model and some applications. Theoretical Econ. 9, 695-752.

Lin, Xu, 2010. Identifying Peer effects in student academic achievements by spatial autoregressive models with group unobservables. J. Lab. Econ. 28 (4), $825-860$.

Liu, Xiaodong, Pattachini, Eleanora, Zenou, Yves, Lee, Lung-Fei, 2014. Criminal networks: who is the key player? Working paper, Stockholm University.

Milgrom, Paul, Roberts, John, 1990. Rationalizability, learning and equilibrium in games with strategic complementarities. Econometrica 58 (6), $1255-1277$.

Tamer, E.T., 2003. Incomplete bivariate discrete response model with multiple equilibria. Rev. Econ. Stud. 70, $147-167$.

Vives, Xavier, 1990. Nash equilibrium with strategic complementarities. J. Math. Econ. 19, 305-321.

\footnotetext{
16 There are various possible empirical specifications for the $a$ 's (including individual covariates and, possibly, contextual peer effects), the g's (linear-insum or linear-in-means), and the error terms $\varepsilon$. See, for instance, Blume et al. (2010), Bramoullé et al. (2009), De Giorgi et al. (2010), Lin (2010) and Liu et al. (2014).
} 